\title{
Dissipation-induced quantum phase transition in a quantum box
}

\author{
László Borda ${ }^{1,2}$, Gergely Zaránd ${ }^{1}$ and Pascal Simon ${ }^{3}$ \\ 1 Theoretical Physics Department, Institute of Physics, \\ Budapest University of Technology and Economics, Budafoki út 8, H-1521 \\ ${ }^{2}$ Research Group of Hungarian Academy of Sciences, Budafoki út 8, Budapest, H-1521 \\ ${ }^{3}$ Laboratoire de Physique et Modélisation des Milieux Condensés, \\ CNRS et Université Joseph Fourier, 38042 Grenoble, France
}

(Dated: June 23, 2018)

\begin{abstract}
In a recent work, Le Hur has shown that dissipative coupling to gate electrodes may play an important role in a quantum box near its degeneracy point [K. Le Hur, Phys. Rev. Lett. 92, 196804 (2004)]: While quantum fluctuations of the charge of the dot tend to round Coulomb blockade charging steps of the box, strong enough dissipation suppresses these fluctuations and leads to the reappearance of sharp charging steps. In the present paper we study this quantum phase transition in detail using bosonization and numerical renormalization group methods in the limit of vanishing level spacing.
\end{abstract}

PACS numbers: 75.20.Hr, 71.27.+a, 72.15.Qm

\section{INTRODUCTION}

Coulomb blockade is one of the most studied and most basic correlation effects that occurs in mesoscopic devices: $\underline{\underline{1}}$ This phenomenon appears in small structures (quantum dots or metallic islands) weakly connected to the rest of the world. The capacitance of these small devices can be very small and therefore the charging energy $E_{C}$, corresponding to the energy cost of putting an extra electron on the device, can become large. If this charging energy is larger than the measurement temperature then correlation effects can become important, thermal fluctuations of the charge of the island become small, and transport through the device is typically suppressed as we lower the temperature.

In addition to the above-mentioned thermal fluctuations, Coulomb blockade can also be lifted by quantum fluctuations. There is two important known mechanisms that lead to quantum fluctuations: (a) Depending on the applied gate voltages, two charging states of the quantum dot can be degenerate, and electrons can thus gain kinetic energy by hybridizing with states in the leads, accompanied by strong quantum fluctuations of the charge. ${ }^{2.3}$ (b) Quantum fluctuations can play an important role at temperatures below the single particle level spacing of the dot too, if the dot contains an odd number of electrons or if the ground state of the isolated dot is degenerate. In this case the system gets rid of the residual entropy associated with the spin degeneracy of the isolated dot through the Kondo effect, $\frac{4}{=}$ which consists of binding conduction electrons in the attached metallic leads antiferromagnetically to the spin of the dot 5.6

The simplest device where charge fluctuations play a dominant role is the single electron box (SEB) schematically shown in Fig. 1. In this device one couples an isolated quantum box capacitively to a gate electrode and to an external lead through a tunnel junction. The isolated box can be very well described by the following simple

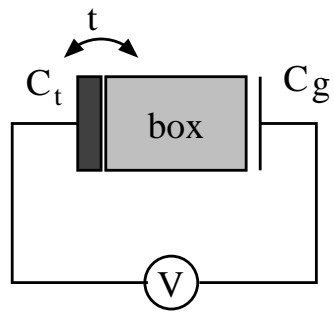

(a)

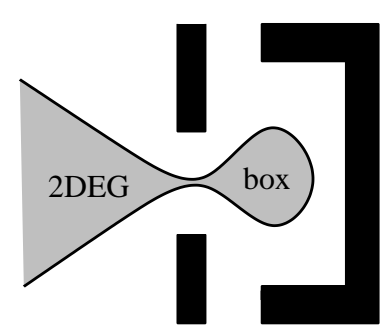

(b)
FIG. 1: Sketch of the single electron box. Fig. b. shows the top view of the regime where electrons move in the the two-dimensional electron gas. Black areas indicate various electrodes necessary to shape the electron gas.

Hamiltonian ${ }^{\underline{1}}$

$$
H_{\mathrm{box}}=\sum_{n, \sigma} \epsilon_{n} d_{n, \sigma}^{\dagger} d_{n, \sigma}+\frac{e^{2}}{2 C}\left(\hat{N}-n_{g}\right)^{2}
$$

where $d_{n, \sigma}^{\dagger}$ creates an electron on the dot with spin $\sigma$ in a single particle state of energy $\epsilon_{n}$. The second term accounts for the electron-electron interaction with $C$ the total capacitance of the box, and $\hat{N}=\sum_{n, \sigma}: d_{n \sigma}^{\dagger} d_{n, \sigma}$ : the number of extra electrons on the island. In Eq. (1) $n_{g}=V_{g} C_{g} / e$ stands the dimensionless gate voltage expressed in terms of the gate voltage $V_{g}$, the gate capacitance, $C_{g}$, and the electron charge $e$. Clearly, the number of electrons on an isolated box jumps by one at approximately half-integer values of $n_{g}$ as we increase $n_{g}$.

In reality, however, the box is not fully isolated from the rest of the world, and in the vicinity of these degeneracy points quantum fluctuations to the leads need to be considered. In the following we shall restrict our considerations to the particularly interesting regime where the temperature is much larger than the typical single particle level spacing $\Delta$ on the box, but is also much smaller 


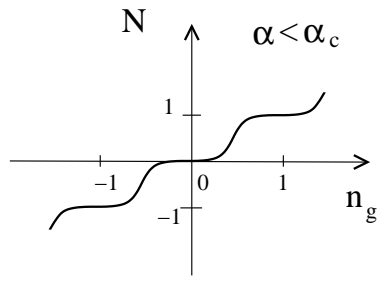

(a)

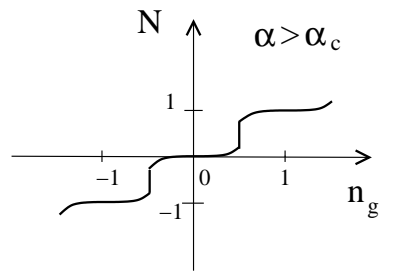

(b)
FIG. 2: The number of electrons on a single electron box as a function of the dimensionless gate voltage. (a) The sudden jumps of an isolated box become smeared out due to quantum fluctuation as soon as we couple the box to a lead. (b) Large enough dissipation restores the discrete jumps.

than the charging energy $E_{C}=e^{2} / 2 C$,

$$
\Delta \ll T \ll E_{C} .
$$

Furthermore, we shall focus our attention to the vicinity of the above degeneracy points, $n_{g} \approx$ half-integer. As shown by Matveev, ${ }^{2}$ in this regime quantum fluctuations of the charge on the dot can be treated by mapping the Hamiltonian to that of the anisotropic two-channel Kondo problem. The finite jumps in $\langle\hat{N}\rangle$ are rounded by these quantum fluctuations, and all what is left from them in the $\Delta \rightarrow 0$ limit is a logarithmic divergence in the slope of $\langle\hat{N}\rangle\left(n_{g}\right)$ at the degeneracy points $n_{g} \approx$ halfinteger (see Fig. [2]a).

However, as shown recently by Le Hur, this picture is not complete ${ }^{9}$ In Eq. (1) one typically assumes that the gate voltage is constant. In reality, however, this gate voltage represents electromagnetic degrees of freedom. The fluctuations of these electromagnetic degrees of freedom must be considered and typically result in Ohmic dissipation, characterized by a dimensionless dissipation strength $\alpha \stackrel{10}{\underline{\underline{10}}}$ The specific value of this dissipation depends on the resistance $R_{g}$ of the gate electrode and the gate capacitance, and is proportional to

$$
\alpha \approx \frac{C_{g}^{2}}{4 C^{2}} \frac{R_{g}}{R_{Q}},
$$

with $R_{Q}=\frac{h}{2 e^{2}}$ the quantum resistance unit. As shown by Le Hur, this additional dissipative coupling leads to a quantum phase transition: Below a critical dissipation strength, $\alpha_{c}$, dissipation is ultimately irrelevant, and the scenario of Matveev holds with somewhat modified parameters. If, however, $\alpha$ is large enough then dissipation dominates, charge fluctuations are suppressed, and $\langle\hat{N}\rangle\left(n_{g}\right)$ displays finite jumps at half-integer values of $n_{g}$ at $T=0$ temperature, as shown in Fig. 2 b $\frac{9}{2}$ This critical value of dissipation depends on the dimensionless conductance $g$ of the tunnel junction, $\alpha_{c}=\alpha_{c}(g)$.

We have to remark here that the above considerations hold only in the limit of vanishing level spacing, $\Delta \rightarrow 0$. In reality, level spacing on the dot is finite, and for semiconducting devices the ratio $E_{C} / \Delta$ cannot be much larger than about $100, \stackrel{11,12}{1}$ Thus the level spacing $\Delta$ ultimately provides a finite cut-off similar to a finite temperature, and the slope of $\langle\hat{N}\rangle\left(n_{g}\right)$ is expected to remain finite even at $T=0$ temperature. Although it is not clear what happens below the level spacing, in a real system the phase transition above is thus probably reduced to a cross-over (see our conclusions too).

In the present paper we shall investigate this phase transition employing non-perturbative methods such as Abelian bosonization ${ }^{14}$ and numerical renormalization group to a somewhat generalized version of the quantum box model ${ }^{20.21}$ These methods enable us to obtain the full phase diagram of the quantum box.

Using the bosonization approach we are able to construct the non-perturbative scaling equations that describe the above quantum phase transition. At the degeneracy point, we find a Kosterlitz-Thouless quantum phase transition, in agreement with the weak coupling result of Le Hur $\stackrel{9}{-}$ We also determine the scaling of the Kondo temperature $T_{K}$ associated with the charge fluctuations non-perturbatively. Apart from an overall prefactor, this scale vanishes as

$$
T_{K}(\alpha) \approx E_{C} \exp \left\{-\frac{\pi}{\sqrt{\frac{2}{\pi} \sqrt{g}\left(1-\alpha_{c}\right)^{1 / 2}} \sqrt{\alpha_{c}-\alpha}}\right\}
$$

where $g=\frac{h}{2 e^{2}} G$ is the dimensionless conductance of the junction. Thus $T_{K}$ approaches zero very fast but continuously as one approaches the critical value $\alpha_{c}$ from below.

The phase transition also manifests itself in the $T=0$ temperature linear conductance of the tunnel junction, which displays a first order phase transition:

$$
g(T=0, \alpha)=\frac{1}{2} \theta\left(\alpha_{c}-\alpha\right) .
$$

We also study the above phase transition using the powerful method of numerical renormalization group (NRG). Since the calculation for fermions with spin is computationally extremely demanding, we will have to restrict ourself to the case of spinless fermions. The phase transition within this method simply appears as a change in the finite size spectrum. The NRG method also enables us to determine the shape of the step in $\langle\hat{N}\rangle\left(n_{g}\right)$ and $T_{K}$ non-perturbatively, and compute various spectral functions. Our results agree very well with the scenario of Ref. 9 and the bosonization results.

The paper is structured as follows: In Section II we introduce our basic notations and show how to map the problem to a Kondo model. Section [II] is devoted to the construction of the non-perturbative scaling equations and their analysis. The numerical renormalization group results are presented in Section IV Finally, we summarize our results and discuss future perspectives in Section $\nabla$ 


\section{HAMILTONIAN}

We shall assume that coupling between the lead and the dot is small, so that charge fluctuations can be described within the tunneling approximation

$$
H_{\mathrm{tun}}=t \sum_{n, \epsilon, \sigma}\left(d_{n, \sigma}^{\dagger} c_{\epsilon, \sigma}+\text { h.c. }\right)
$$

where the operators $c_{\epsilon, \sigma}^{\dagger}$ create conduction electrons in the lead with energy $\epsilon$ and $\operatorname{spin} \sigma$,

$$
H_{\text {lead }}=\sum_{\epsilon, \sigma} \epsilon c_{\epsilon, \sigma}^{\dagger} c_{\epsilon, \sigma}
$$

In Eq. (6) we assumed that there is only a single tunneling mode that is coupled to the dot electrons so that the tunneling matrix elements can be replaced by a simple number. The situation is much more complex in case of several tunneling modes and shall not be discussed here (see Ref. 12).

Since all half-integer values of $n_{g}$ are essentially equivalent, to obtain Matveev's mapping, we shall focus to the vicinity of the point $n_{g}=1 / 2$. The important quantum fluctuations in this regime are dominated by the two charging states $|N=0\rangle$ and $|N=1\rangle$ of the dot. Other charging states of the dot are separated at least by an energy $\sim E_{C}$, and in the small temperature/tunneling regime their effect is only to slightly renormalize the values of $t$ and $E_{C}$. We can thus restrict our considerations to the charging states $N=0$ and $N=1$, and keep track of charge fluctuations of the box by introducing an orbital pseudospin $T$, the states $T_{z}=(1-\hat{N}) / 2= \pm 1 / 2$ corresponding to states with $N=0,1$. Note that we still have to keep track of internal electron-hole excitations of the box, since our energy scale is larger than the level spacing.

To rewrite the tunneling Hamiltonian in a more suggestive way we introduce the new fields normalized by the density of states in the box and in the lead, $\varrho_{\text {box }}$ and $\varrho_{\text {lead }}$, respectively

$$
D_{\sigma} \equiv \frac{1}{\sqrt{\varrho_{\mathrm{box}}}} \sum_{n} d_{n, \sigma}, \quad C_{\sigma} \equiv \frac{1}{\sqrt{\varrho_{\mathrm{lead}}}} \sum_{\epsilon} d_{\epsilon, \sigma}
$$

and organize them into a four component spinor

$$
\psi_{\tau, \sigma} \equiv\left(\begin{array}{c}
C_{\sigma} \\
D_{\sigma}
\end{array}\right)
$$

The normalization in Eq. (8) has been chosen is such a way that the imaginary time propagator of the field $\psi$ satisfies $\left\langle\mathrm{T}_{\tau} \psi_{\tau \sigma}(\tau) \psi_{\tau^{\prime} \sigma^{\prime}}^{\dagger}(0)\right\rangle=\delta_{\sigma, \sigma^{\prime}} \delta_{\tau, \tau^{\prime}} / \tau$. In this language we can rewrite the tunneling part of Hamiltonian as

$$
H_{\text {perp }}=\frac{j_{\perp}}{2}\left(T^{+} \psi_{\sigma}^{\dagger} \tau^{-} \psi_{\sigma}+\text { h.c. }\right)
$$

where the operator $\tau^{ \pm}$just flips the orbital spin $\tau$ of the field $\psi_{\tau \sigma}$, and the $j_{\perp}$ is a dimensionless coupling proportional to the tunneling, $j_{\perp}=2 t \sqrt{\varrho_{\text {box }} \varrho_{\text {lead }}}$. Thus $j_{\perp}^{2}$ is directly related to the dimensionless conductance $g$ of the tunnel junction

$$
g=\frac{G}{G_{Q}}=\frac{\pi^{2}}{4} j_{\perp}^{2}
$$

with $G_{Q}=2 e^{2} / h$, the quantum conductance unit. Clearly, Eq. (10) is just the Hamiltonian of an anisotropic two-channel Kondo model, ${ }^{2.13}$ the orbital spins $T$ and $\tau$ playing the role of the spins of the original two-channel Kondo model, and the electron spin $\sigma$ providing the silent channel index. The presence of this additional channel index makes the physics of the two-channel Kondo model entirely different from that of the single channel Kondo problem, and leads to non-Fermi liquid properties, such as the logarithmically divergent capacitance in our case. We emphasize again, that the above mapping holds only in the regime $\Delta<T, \omega, \ldots<E_{C}$, where the level spacing of the box can be neglected.

Deviations from the point $n_{g}=1 / 2$ appear in this language as a local field acting on the orbital spin of the box

$$
\frac{e^{2}}{2 C}\left(\hat{N}-n_{g}\right)^{2} \rightarrow-B T^{z},
$$

where the effective magnetic field is simply given by $B=E_{C}\left(1-2 n_{g}\right)$. It is clear from this expression that fluctuations of the gate voltage, $\delta V_{g}$ will result in a dissipative coupling of the following form

$$
H_{\mathrm{diss}}=\lambda T^{z} \varphi
$$

where for an Ohmic heat bath the bosonic field $\varphi$ decays as

$$
\left\langle\mathrm{T}_{\tau} \varphi(\tau) \varphi(0)\right\rangle=\frac{1}{\tau^{2}}
$$

The dimensionless coupling $\lambda$ above is related to the usual coupling $\alpha$ as $\alpha=\lambda^{2} / 2$. The specific value of $\lambda$ depends on the properties of gate electrodes and is approximately given by Eq. (3). Thus for very resistive gate electrodes, $\alpha$ can be large, while if the gate electrodes are good conductors, then $\alpha$ is small.

Eqs. (10), (12), and (14) constitute the effective Hamiltonian that describes the physics of the dot in the vicinity of the degeneracy point in the presence of dissipation. In the rest of the paper we shall, however, include an additional term in the Hamiltonian of the form

$$
H_{z}=\frac{j_{z}}{2} \sum_{\sigma} T^{z} \psi_{\sigma}^{\dagger} \tau^{z} \psi_{\sigma}
$$

too. This term describes charging state dependent scattering of the conduction electrons. While this term is typically small, it is generated under the renormalization group procedure. Furthermore, in other physical systems such as a Kondo spin coupled to fluctuations of quantum critical spin degrees of freedom, e.g. $\frac{15}{15}$ this term is present in the bare Hamiltonian as well. 


\section{BOSONIZATION}

The non-perturbative method of Abelian bosonization can be very efficiently used to construct the phase diagram of our model, and allows us to treat both the dissipative coupling $\lambda$ and the coupling $j_{z}$ non-perturbatively. For the sake of simplicity, we shall first discuss the somewhat simpler case of spinless Fermions and then restore spin indices. Throughout this Section we shall use the bosonization scheme of Ref. 14 .

\section{A. Bosonization for spinless Fermions}

For spinless fermions the field $\psi$ has only two orbital indices, $\tau= \pm$. Correspondingly, we have to introduce two left-moving bosonic fields $\Phi_{ \pm}$to represent $\psi_{ \pm}, 14$

$$
\psi_{ \pm}=F_{ \pm} \frac{1}{\sqrt{a}} e^{-i \Phi_{ \pm}}
$$

where $F_{ \pm}$denotes the Klein factor, and $a$ is a short distance cut-off $\sim v_{F} / E_{C}$. In terms of these bosonic fields the interaction part of the Hamiltonian reads

$$
\begin{aligned}
H_{z} & =\frac{\tilde{j}_{z}}{\sqrt{2}} T^{z} \partial_{x} \Phi_{T} \\
H_{\text {perp }} & =j_{\perp} \frac{1}{2 a}\left(T^{+} e^{-i \sqrt{2} \Phi_{T}}+\text { h.c. }\right)
\end{aligned}
$$

where for the sake of simplicity we suppressed Klein factors and introduced the orbital field, $\Phi_{T}=\left(\Phi_{+}-\right.$ $\left.\Phi_{-}\right) / \sqrt{2}$. Note that the charge field $\Phi_{C}=\left(\Phi_{+}+\Phi_{-}\right) / \sqrt{2}$ completely decouples from the orbital spin of the box. Particular care is needed while deriving Eq. (17): Introducing the bosonization cut-off scheme renormalizes the coupling constants, $j_{z} \rightarrow \tilde{j}_{z}$.

$$
\tilde{j}_{z}=\frac{4 \delta_{z}}{\pi}=\frac{4}{\pi} \operatorname{atan}\left(\frac{\pi j_{z}}{4}\right)
$$

with $\delta_{z}$ the phase shift generated by $j_{z}$. Representing the field $\varphi$ as the derivative of another bosonic field $\Phi$ we can finally rewrite the dissipative term as

$$
H_{\mathrm{diss}}=\lambda T^{z} \partial_{x} \Phi
$$

In this bosonized form the non-interacting part of the Hamiltonian becomes

$$
H_{0}=\int \frac{d x}{4 \pi}\left[\sum_{\mu=T, C}:\left(\partial_{x} \Phi_{\mu}\right)^{2}:+:\left(\partial_{x} \Phi\right)^{2}:\right]
$$

where the first two terms represent electron-hole excitations on the dot and in the lead, and the last term gives the energy of excitations responsible for dissipation.

To obtain the phase diagram of our model we carried out a non-perturbative scaling analysis. As a first step we eliminated the terms proportional to $\lambda$ and $j_{z}$ by applying a unitary transformation

$$
U=\exp \left\{i T_{z}\left(\lambda \Phi(0)+\frac{4 \delta_{z}}{\pi \sqrt{2}} \Phi_{T}(0)\right)\right\} .
$$

This unitary transformation brings $H_{\text {perp }}$ to the following form

$$
H_{\text {perp }}=\frac{j_{\perp}}{2 a}\left[T^{+} e^{i \lambda \Phi(0)+i\left(\frac{4 \delta_{z}}{\pi \sqrt{2}}-\sqrt{2}\right) \Phi_{T}(0)}+\text { h.c. }\right] .
$$

The scaling dimension of $j_{\perp}$ can be read out of this equation and one obtains

$$
\frac{d j_{\perp}}{d l}=\left[4 \frac{\delta_{z}}{\pi}-4\left(\frac{\delta_{z}}{\pi}\right)^{2}-\alpha\right] j_{\perp}+\mathcal{O}\left(j_{\perp}^{3}\right),
$$

where $\alpha=\lambda^{2} / 2$ is the dissipation strength, and $l=\ln a$ is the scaling variable. The first term in this equation is just the famous non-perturbative equation of Yuval and Anderson. 16 The second term in this scaling equation just means that dissipative coupling to charge fluctuations on the gate tends to suppress quantum fluctuations of the charge of the dot. It is this term that drives the phase transition.

The scaling equations of $\alpha$ and $\delta_{z}$ can be simply obtained by taking the operator product expansion of the term $j_{\perp}$ with itself. In this way we obtain:

$$
\begin{aligned}
& \frac{d}{d l}\left(\frac{4 \delta_{z}}{\pi}\right)=\left(1-\frac{2 \delta_{z}}{\pi}\right) j_{\perp}^{2}, \\
& \frac{d \alpha}{d l}=-\alpha j_{\perp}^{2},
\end{aligned}
$$

where terms of order $j_{\perp}^{3}$ have been dropped. The first of these equations has been derived by Yuval and Anderson, 16 and shows that $j_{\perp}$ tends to boost up $\delta_{z}$ and generate a Kondo effect. The second equation shows that quantum fluctuations suppress dissipation effects. There is thus a competition between $j_{\perp}$ and $\alpha$, which mutually tend to suppress each-other.

Depending on the model parameters, we have to distinguish two regimes: In the small dissipation regime dissipation is irrelevant, $\alpha$ scales to zero, and charge fluctuations generated by $j_{\perp} \sim t$ start to dominate at an energy scale $T_{K}$, the so-called Kondo energy. This Kondo fixed point corresponds to $\delta_{z}=\pi / 2$ and $j_{\perp} \rightarrow \mathcal{O}(1){ }^{16}$ It is easy to show that the dissipative coupling is indeed irrelevant at this Kondo fixed point: This follows from the observation that the Kondo fixed point is described by a Fermi liquid theory. $\frac{4}{4}$ s a consequence, the orbital susceptibility is finite at zero temperature, $\chi_{T} \sim 1 / T_{K}$, and correspondingly the imaginary time correlation function of $T_{z}$ decays as $\left\langle\mathrm{T}_{\tau} T_{z}(\tau) T_{z}(0)\right\rangle \sim 1 / \tau^{2}$. Therefore the correlation function $\left\langle\mathrm{T}_{\tau} T_{z}(\tau) \varphi(\tau) T_{z}(0) \varphi(0)\right\rangle$ decays as $1 / \tau^{4}$, implying that the scaling dimension of the dissipation is -1 at the Kondo fixed point, and is therefore irrelevant 17 The capacitance of the dot for these small values of $\alpha$ is simply proportional to the orbital susceptibility, $C \sim \chi_{T} \sim 1 / T_{K}$. 
If, on the other hand, the bare value of $\alpha$ is larger than a critical value, $\alpha_{c}\left(j_{\perp}, \delta_{z}\right)$, then quantum fluctuations are suppressed, and $j_{\perp}$ scales to 0 implying that charge fluctuations are suppressed. This phase can be called localized in the sense that if we prepare the box in the state $\hat{N}=0$ at the degeneracy point, then the expectation value of $\hat{N}$ will never approach its equilibrium value $1 / 2$,

$$
\lim _{t \rightarrow \infty}\langle\hat{N}(t)\rangle<1 / 2 \quad\left(\alpha>\alpha_{c}\right) .
$$

This behavior is analogous to that of the dissipative twostate system 10

It is easy to determine this critical value of $\alpha$ in the $j_{\perp} \rightarrow 0$ limit from our scaling equations:

$$
\alpha_{c}\left(j_{\perp} \rightarrow 0\right)=4 \frac{\delta_{z}}{\pi}\left(1-\frac{\delta_{z}}{\pi}\right) .
$$

To obtain the phase diagram in the vicinity of this line. we rewrite the scaling equations as

$$
\begin{aligned}
& \frac{d j_{\perp}}{d l} \approx j_{\perp} r, \\
& \frac{d r}{d l}=j_{\perp}^{2},
\end{aligned}
$$

where we introduced the variable

$$
r=4 \frac{\delta_{z}}{\pi}\left(1-\frac{\delta_{z}}{\pi}\right)-\alpha,
$$

measuring the distance from the line Eq. (27). These equations clearly describe a Kosterlitz-Thouless phase transition 18 Solving these equations we find that for small values of $j_{\perp}$

$$
\alpha_{c} \approx\left|j_{\perp}\right|+4 \frac{\delta_{z}}{\pi}\left(1-\frac{\delta_{z}}{\pi}\right)
$$

and the corresponding Kondo scale vanishes as one approaches $\alpha$ as

$$
T_{K} \approx E_{C} \exp \left\{-\frac{\pi}{\sqrt{2\left|j_{\perp}\right|} \sqrt{\alpha_{c}-\alpha}}\right\} .
$$

It is important to remark at this point that the above scaling equations describe the nature of the phase transition even for large values of $j_{\perp}$. The reason is that in the vicinity of the quantum phase transition all scaling trajectories approach the critical line defined by $j_{\perp}=0$ and $\alpha=4 \frac{\delta_{z}}{\pi}\left[1-\frac{2 \delta_{z}}{\pi}\right]$. Thus the initial part of the scaling just renormalizes the effective value of the various couplings that enter Eq. (40), but the overall picture does not change.

\section{B. Fermions with spin}

The considerations of the previous subsection can be easily generalized to the case of Fermions with spin. The scaling equation of $j_{\perp}$ then becomes

$$
\frac{d j_{\perp}}{d l}=\left[4 \frac{\delta_{z}}{\pi}-8\left(\frac{\delta_{z}}{\pi}\right)^{2}-\alpha\right] j_{\perp},
$$

while the other two scaling equations read

$$
\begin{aligned}
& \frac{d}{d l}\left(\frac{4 \delta_{z}}{\pi}\right)=\left(1-\frac{4 \delta_{z}}{\pi}\right) j_{\perp}^{2}, \\
& \frac{d \alpha}{d l}=-\alpha j_{\perp}^{2} .
\end{aligned}
$$

In the absence of dissipation, these scaling equations have been derived first by Vladár, Zimányi and Zawadowski $\stackrel{19}{ }$ Similar to the spinless case, two regimes can be distinguished $\stackrel{9}{9}^{-}$For small dissipations $\alpha$ is irrelevant, and at the degeneracy point of the dot the couplings $j_{\perp}$ and $\delta_{z}$ flow to the two-channel Kondo fixed point, $\delta_{z}=\pi / 4, j_{\perp} \rightarrow \mathcal{O}(1)$. The physics is, however, not that of a Fermi liquid due to the presence of electron spin: the orbital susceptibility (i.e. the capacitance) of the dot diverges logarithmically below the Kondo temperature, $\chi_{T} \sim \ln \left(T_{K} / T\right) / T_{K}{ }^{2,13}$ This logarithmic divergence indicates that the deviation from the degeneracy point $n_{g}=1 / 2$ represent a relevant perturbation of scaling dimension $1 / 2$, and as a consequence, the slope of $\langle\hat{N}\rangle\left(n_{g}\right)$ diverges logarithmically at $T=0$ temperature as one approaches $n_{g}=1 / 2$ (if we neglect the finite level spacing on the dot).

It is easy to check that dissipation is irrelevant at this two-channel Kondo fixed point too: The logarithmic divergence of the orbital susceptibility corresponds to an asymptotic decay $\left\langle\mathrm{T}_{\tau} T_{z}(\tau) T_{z}(0)\right\rangle \sim 1 / \tau$. Consequently, the correlation function $\left\langle\mathrm{T}_{\tau} T_{z}(\tau) \varphi(\tau) T_{z}(0) \varphi(0)\right\rangle$ decays as $1 / \tau^{3}$ at the two-channel Kondo fixed point, implying that the scaling dimension of the dissipation is $-1 / 2$ at the Kondo fixed point, and is therefore irrelevant $\underline{17}$

If, however, $\alpha$ is large enough then again, the dissipation drives $j_{\perp}$ to zero, and we recover the localized phase with jumps in the function $\langle\hat{N}\rangle\left(n_{g}\right)$. Similar to the spinless case, we can determine the boundary between these two phases by introducing the variable

$$
r=4 \frac{\delta_{z}}{\pi}\left(1-\frac{2 \delta_{z}}{\pi}\right)-\alpha
$$

and rewriting the scaling equations in terms of it. Keeping only the lowest order terms in $r$ and $j_{\perp}$ we obtain again a set of two coupled differential equations describing a Kosterlitz-Thouless phase transition:

$$
\begin{aligned}
& \frac{d j_{\perp}}{d l} \approx j_{\perp} r, \\
& \frac{d r}{d l}=(1-\alpha) j_{\perp}^{2} .
\end{aligned}
$$

For small values of $j_{\perp}$ the phase boundary is thus given by

$$
\alpha_{c}^{\text {spin }} \approx j_{\perp}+4 \frac{\delta_{z}}{\pi}\left(1-\frac{2 \delta_{z}}{\pi}\right),
$$

and the Kondo scale vanishes as one approaches $\alpha_{c}$ from below as

$$
T_{K} \approx E_{C} \exp \left\{-\frac{\pi}{\sqrt{2\left|j_{\perp}\right|\left(1-\alpha_{c}^{\text {spin }}\right)^{1 / 2}} \sqrt{\alpha_{c}^{\text {spin }}-\alpha}}\right\} .
$$




\section{NUMERICAL RENORMALIZATION GROUP CALCULATION}

In order to obtain results for different physical quantities we performed Wilson's numerical renormalization group method (NRG) on the model ${ }^{20.21 .22 .23}$ This method is especially suitable for quantum impurity problems and can be used to determine dynamical as well as thermodynamic properties with high accuracy. However, performing such a calculation on the Bose-Fermi Kondo model, a conceptual problem arises: Originally, NRG was designed to treat fermionic problems, where the method is based on a logarithmic discretization of the conduction band and then a mapping of it onto a semi-infinite chain. In the present model we need to treat both fermionic and bosonic baths at the same time. Even though the NRG method has recently been extended to bosonic systems $\stackrel{24.25}{,}$ the bosonic calculation needs considerable computational effort. We therefore decided to treat this problem in a different way.

Fortunately, in the present model the bosonic field $\varphi$ is Ohmic. This implies that we can represent this field by coupling $T_{z}$ to the total density of $F$ fermionic fields in the following way:

$$
H_{\text {diss }}=\frac{g}{2} T^{z} \sum_{i=1}^{F} \Psi_{i}^{\dagger} \Psi_{i} .
$$

Here the fields $\Psi_{i}$ denote some fermion fields normalized as $\left\langle\mathrm{T}_{\tau} \Psi_{i}(\tau) \Psi_{j}^{\dagger}(0)\right\rangle=\delta_{i, j} / \tau$. To make this mapping complete, we have to find the relation between $q$ and the dissipation strength $\alpha$. It is a relatively simple matter to show that these are connected as

$$
\alpha=2 F\left(\frac{\delta_{g}}{\pi}\right)^{2},
$$

with $\delta_{g}=\operatorname{atan}(\pi g / 4)$ the phase shift associated with the potential scattering $g$.

The advantage of this trick is that we can use just fermionic fields in our calculations. On the other hand, this trick also implies that all along the calculations, we carry along $F-1$ bosonic fields which are decoupled from $T_{z}$. For large values of $F$ we thus keep track of many redundant degrees of freedom. Furthermore, we cannot reach any value of $\alpha$ in this way. In our calculations, e.g., we used $F=2$ fermion fields allowing to map out the phase diagram for $0 \leq \alpha \leq 1$.

It is clear from the previous subsection that to represent the physical system considered in the present paper, one needs at least three bands of spinful fermions: one of them, $\Psi_{\sigma}$ would be used to represent dissipative term in the Hamiltonian while two others are just the electronic degrees of freedom, $\psi_{\tau \sigma}$. This implies that one needs a three-channel NRG code to attack the problem in its full glory. Unfortunately, we do not have too many symmetries simplifying our problem: Throughout the calculation we used an $\mathrm{SU}(2)$ symmetry associated with particlehole symmetry and a U(1) symmetry associated with the

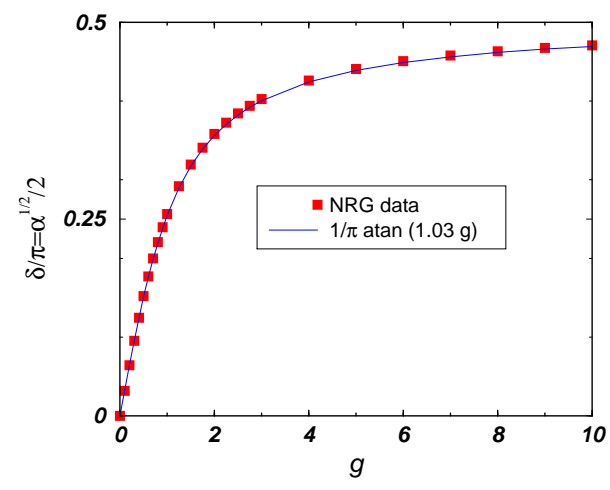

FIG. 3: (color online) Relation between the phase shift $\delta_{g}$ extracted from the finite size spectrum and the fermionic coupling $g$. The continuous line shows the fit with Eq. (43).

$z$-component of the orbital spin. As a consequence, we were able to study only the case of spinless fermions with sufficient accuracy. Fortunately, as shown in the previous section, most features of the spinful case already appear in the spinless version of the model. Furthermore, the spinless case is also of relevance: this is realized in a single electron box by applying an external magnetic field that lifts the electron-spin degeneracy and drives the system away from the unstable two-channel Kondo fixed point.

The very first step is to calibrate the method, i.e., to determine the correspondence between the fermionic coupling $g$ and $\alpha$. In principle, these are related by Eq. (42). However, the NRG discretization renormalizes the effective value of $g$. To do that, we have performed calculations for $j_{\perp}=j_{z}=0$ in the presence of a term $\Delta_{0} T^{x}$, i.e., for the spin-boson problem, and extracted the phase shifts from the finite size spectra. In that way we found that the phase shifts can be expressed with very high accuracy as

$$
\delta_{g}=\operatorname{atan}(f(\Lambda) g),
$$

where $\Lambda$ is the parameter of the logarithmic discretization used in NRG and $f(\Lambda)$ is a factor close to unity that must be determined numerically. In particular, for $\Lambda=2$ used throughout this paper we find $f(\Lambda=2)=1.03$. The phase shift above can then be plugged into Eq. (42) to obtain the corresponding value of $\alpha$. This procedure is illustrated in Fig 3 A similar expression gives the value of $\delta_{z}$ as a function of $j_{z}$.

The most basic result one can obtain by NRG is the finite size spectrum, i.e., the rescaled lowest lying energy levels plotted against the iteration number. We show two

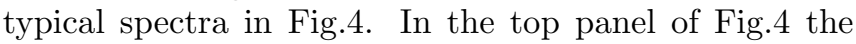
spectrum crosses over to that of the usual Kondo fixed point ${ }^{20}$ with uniform level spacing at around $N \sim 60$. In the bottom panel, however, no such crossover occurs, at least not before the 80th iteration indicating that the Kondo effect is suppressed by the strong coupling to the bosonic bath which tends to suppress charge fluctuations 

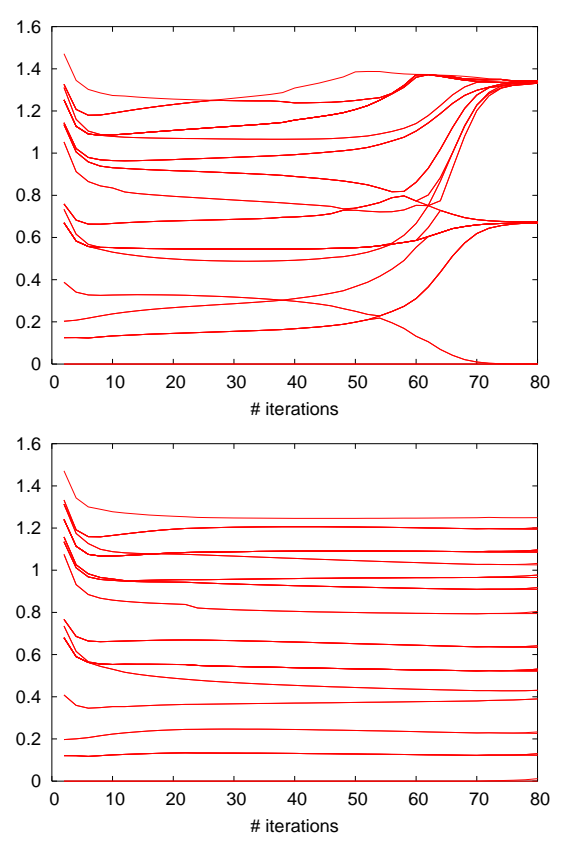

FIG. 4: (color online) Finite size spectra obtained by NRG for the two distinct regimes. The upper part shows the spectrum for $\alpha=0.653, j_{z}=0.2, j_{\perp}=0.7$, while the lower part stands for the same $j$ s but for $\alpha=0.667$. It is transparent that while on the left figure the levels crosses over to the wellknown Kondo spectrum, on the right panel no such crossover occurs.

and thus localizes the orbital spin of the dot. This robust difference in the finite size spectra allows us to extract the critical value of the bosonic coupling $\alpha_{c}$ and map out the phase boundary for all values of $j_{\perp}$ and $\delta_{z}$. Numerically we say that we are in the localized (dissipation dominated) phase if the crossover to the Kondo spectrum does not occur within $N=80$ iterations. The critical surface corresponding to this phase transition is shown in Fig.(15).

The case $j_{z}=0$ is of particular interest, since this corresponds to the charge fluctuations at the degeneracy point of the quantum dot. In agreement with Eq. (31) $\alpha_{c}$ increases approximately linearly with $j_{\perp} \sim t \sim \sqrt{g}$ see Fig. [6). In Fig. 6 we also show the phase boundary as a function of $\delta_{z} / \pi$. The results compare very well with Eq. (31).

The dissipation dependence of the Kondo temperature can be also easily extracted from the finite size spectrum. In Fig. 7 we show $1 / \ln \left(E_{C} / T_{K}\right)$ as a function of $\alpha$. According to Eq.( 32$)$ this should vanish as $\sim \sqrt{\alpha_{c}-\alpha}$ as one approaches the phase boundary, implying an extremely fast change in $T_{K}$ as one approaches $\alpha_{c}$. Though it is very difficult numerically to get to the real scaling regime, the obtained results are quite consistent with the analytical expression Eq. (32).

To compute the full shape of the $\langle\hat{N}\rangle\left(n_{g}\right)$ curve, one has to study the model for gate voltages $n_{g} \neq 1 / 2$, which corresponds to a field $B=\left(1-2 n_{g}\right) E_{C}$ applied to the

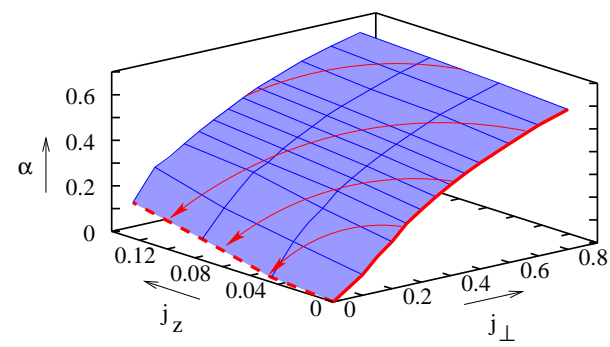

FIG. 5: (color online) The critical surface in the parameter space separating the Kondo regime from the localized regime. The heavy continuous line indicates the phase transition line for the case relevant for the single electron box $j_{z}=0$. The heavy dashed line indicates the critical line, $j_{\perp}=0$. The arrows show the scaling trajectories for $\alpha=\alpha_{c}$. All these critical scaling tranjectories end up at the critical line.
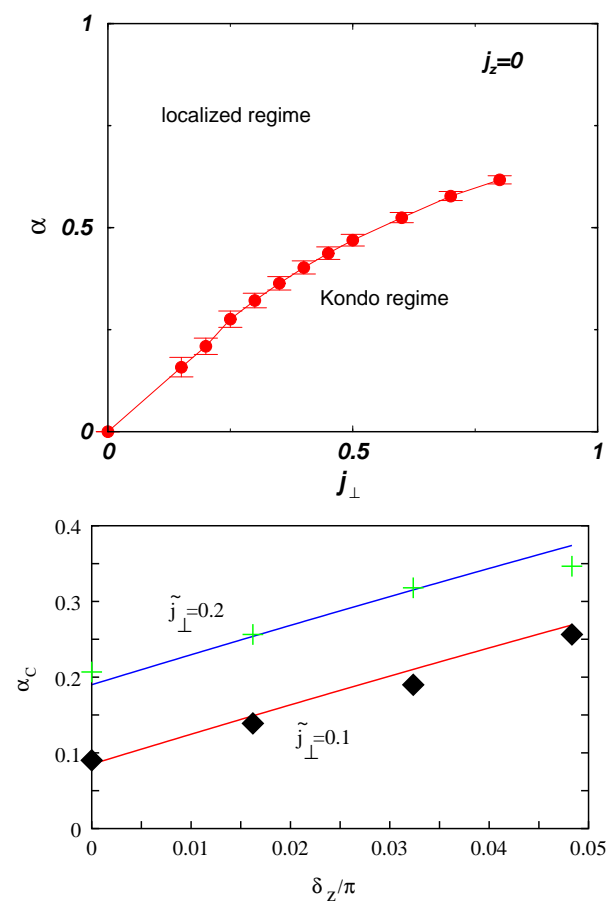

FIG. 6: Top: (color online) The physically relevant $j_{z}=0$ cut of the critical surface. Bottom: Phase boundary as a function of $\delta_{z} / \pi$ for two values of $j_{\perp}$. These phase boundaries compare very well with the analytical expression Eq. (31).

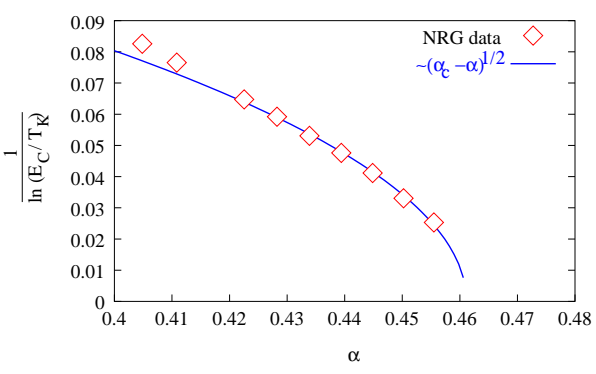

FIG. 7: (color online) Kondo temperature as a function of dissipation, for $j_{\perp}=0.4$ and $j_{z}=0.1$ 


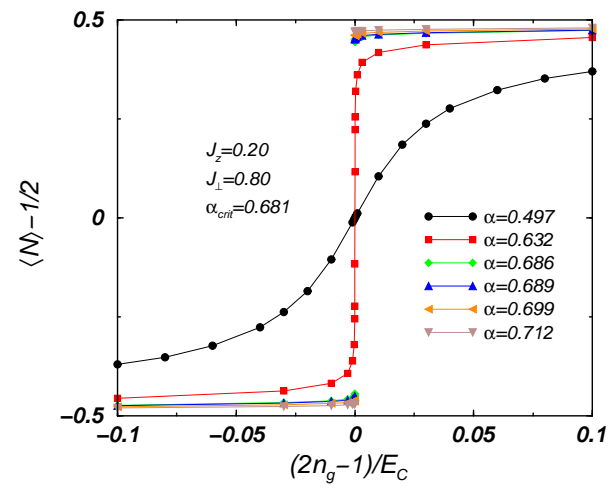

FIG. 8: (color online) The expectation value of $\hat{N}-1 / 2$ for $j_{z}=0.2, j_{\perp}=0.8$ and different values of $\alpha$. For moderate values of the bosonic coupling the step is smeared out by quantum fluctuations of the charge of the dot. The step gets sharper and sharper as $\alpha$ approaches $\alpha_{c}$, and For $\alpha>\alpha_{c}$ a jump appears in $\langle\hat{N}\rangle$.

orbital spin $T_{z}=(1-\hat{N}) / 2$ within our approach. The result is shown in Fig 8 Clearly, for $\alpha<\alpha_{c}$ the Kondo correlations smear out the charging step of the grain.

The Kondo temperature decreases as we increase the coupling of the spin to the bosonic environment, and results in the sharpening of the step in $\langle\hat{N}\rangle$. If we increase the bosonic coupling further, the Kondo effect disappears and $\langle\hat{N}\rangle$ jumps at $n_{g}=1 / 2$. It is difficult to tell from the numerics if the jump remains finite as $\alpha$ approaches $\alpha_{c}$ from above.

However, since the phase transition is of KosterlitzThouless type, one expects that the 'order parameter', i.e. the size of the jump approaches a constant as $\alpha$ approaches $\alpha_{c}$ from the dissipation-dominated (localized) side ${ }^{9}$.

The NRG method also enables us to compute local correlation functions, directly related to measurable quantities. To determine the scattering properties of the conduction electrons, we determined the conduction electrons' $T$-matrix. In the present case, the imaginary part of this quantity is related to the renormalized conductance of the box-lead junction. The computation of this $T$-matrix can be reduced to the determination of the socalled 'composite Fermions' spectral function that can be computed by NRG similar to the single channel Kondo problem, 27,28

$$
\operatorname{Im} T_{\mu}(\omega)=A \sum_{n}\left|\left\langle n\left|F_{\mu}^{\dagger}\right| 0\right\rangle\right|^{2} \delta\left(\omega-\left(E_{n}-E_{0}\right)\right),
$$

where the composite fermion operator is given as

$$
F_{\mu}^{\dagger}=\sum_{k \mu} \vec{S} \vec{\sigma}_{\mu \nu} c_{k \nu}^{\dagger}
$$

The constant $A$ in Eq. (44) can be determined from the condition, $\operatorname{Im} T_{\mu}(\omega)=2 \sin ^{2}(\delta / \pi)$, where $\delta$ denotes the phase shift associated with the fixed point spectrum, and

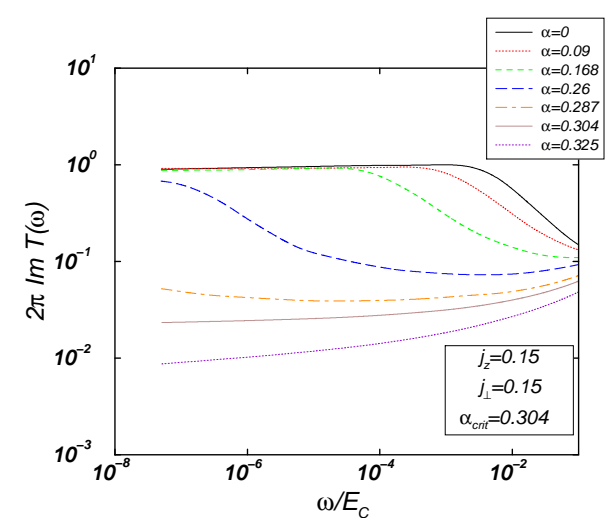

FIG. 9: (color online) The imaginary part of the fermions' $T$ matrix for $j_{z}=0.15, j_{\perp}=0.15$ and for different values of the bosonic coupling. For decoupled bosonic heat bath, the usual Kondo resonance shows up. As $\alpha$ is increased, the width of the Kondo resonance is decreasing and at the critical bosonic coupling $\left(\alpha_{c}=0.304\right)$ it disappears and the electrons become decoupled from the spin.

is $\delta=\pi / 2$ at the quantum fluctuation dominated Kondo fixed point.

The results are shown in Fig 9] These results show that in the fermionic phase the dot-lead junction opens up, and the transmission between the box and the lead becomes perfect at $T=0$ temperature. It is adequate to mention here that the $T$-matrix computed here is much less accurate than that of the one channel Kondo model. The reason is that due to the much bigger Hilbert space we lose a substantial part of the spectral weight.

\section{CONCLUSIONS}

In the present paper we studied the dissipation-induced phase transition at the degeneracy point of a single electron box, discovered by Le Hur, using non-perturbative methods such as bosonization and numerical renormalization group (NRG). Throughout this paper we focused to the limit where the level spacing of the box is negligible. Our calculations confirm the picture of Ref. 9: For small dissipation the dissipative coupling is irrelevant and charging steps of the isolated dot become rounded. However, above a critical value the dissipative coupling caused by fluctuations of the electromagnetic field dominates, and suppresses quantum fluctuations of the charge on the dot. As a results, the charging steps are restored for large enough dissipation.

Our non-perturbative analysis reveals that - as already found by the perturbative analysis of Ref. 9 - this phase transition is of Kosterlitz-Thouless type. Consequently, the Kondo energy, where quantum fluctuations start to dominate goes to zero exponentially fast as $\alpha$ approaches its critical value $\alpha_{c}$.

This phase transition also shows up in the conductance 
of the junction, which has a jump. This can be seen as follows. For $\alpha>\alpha_{c}$ the scaling equations imply that $j_{\perp}$ scales to 0 . Since the linear conductance between the lead and the box is proportional to $j_{\perp}^{2} \sim t^{2}$, this implies that the liner conductance for $\alpha>\alpha_{c}$ is identically zero. For $\alpha<\alpha_{c}$, on the other hand, the system flows to the Kondo (two-channel Kondo) fixed point, where the conductance is $g=1$ both in the spinless and in the spinful case. As a result, the $T=0$ temperature conductance has a jump:

$$
g(T=0, \alpha)=\frac{1}{2} \theta\left(\alpha_{c}-\alpha\right) .
$$

We can also determine the conductance at the critical value of $\alpha$ as a function of temperature from our nonperturbative equations. From the scaling equations we obtain that at $\alpha=\alpha_{c}$ the coupling $j_{\perp}$ scales to zero approximately as

$$
j_{\perp}(T) \approx \frac{1}{\ln \left(T^{*} / T\right)}, \quad\left(\alpha=\alpha_{c}\right)
$$

where $T^{*}$ is a dynamical scale. As a result, the box-lead conductance scales to zero as

$$
g(T) \sim \frac{1}{\ln ^{2} T^{*} / T} \cdot \quad\left(\alpha=\alpha_{c}\right)
$$

The NRG method makes us possible to determine the phase boundary for the case of spinless Fermions between the two phases non-perturbatively. For small values of the tunneling we find from the scaling analysis that

$$
\alpha_{c} \approx j_{\perp},
$$

which agrees very well with the NRG results. This condition translates to the following condition for the device

$$
\frac{C_{g}^{2}}{4 C^{2}} \frac{R_{g}}{R_{Q}} \approx \frac{2}{\pi} \sqrt{g}
$$

where $g$ is the dimensionless conductance of the junction, $C_{g}$ is the gate capacitance, $C$ is the total capacitance of the island, and $R_{g}$ is the resistivity of the gate electrode. In other words, the above phase transition can be reached by increasing the resistance of the gate electrode or by changing the box-lead conductance.

Let us finally make a remark on the finite level spacing $\Delta$. All considerations above hold only in the limit of vanishing level spacing. In reality, however, this level spacing is finite, and plays a role similar to a finite temperature. As a consequence, quantum fluctuations remain always finite at the degeneracy point, and it is not clear if a real phase transition occurs even in the presence of a finite level spacing.

We would like to thank K. Le Hur for valuable discussions. This research has been supported by NSF-MTAOTKA Grant No. INT-0130446, Hungarian Grants No. OTKA T038162, T046267, D048665, and T046303, and the European 'Spintronics' RTN HPRN-CT-2002-00302.
1 R. Wilkins, E. Ben-Jacob, and R. C. Jaklevic, Phys. Rev. Lett. 63, 801 (1989).

2 K.A. Matveev, Zh. Eksp. Teor. Fiz. 98, 1598 (1990) [Sov. Phys. JETP 72, 892 (1991)]; Phys. Rev. B 51, 1743 (1995).

3 N. Andrei, G.T. Zimanyi and G. Schön, Phys. Rev. B 60, 5125 (R) (1999).

4 A.C. Hewson, The Kondo Problem to Heavy Fermions (Cambridge Un iversity Press, Cambridge, 1993).

5 L. I. Glazman and M. E. Raikh, JETP Lett. 47, 452 (1988); T. K. Ng and P. A. Lee, Phys. Rev. Lett. 61, 1768 (1988).

6 D. Goldhaber-Gordon, H. Shtrikman, D. Mahalu, D. Abusch-Magder, U. Meirav, and M.A. Kastner, Nature (London) 391, 156 (1998)

7 I.L. Aleiner, P.W. Brouwer, L.I. Glazman, Physics Reports 358, 309 (2002)

8 The value of $n_{g}$ where the number of electrons changes is slightly shifted with respect to half-integers due to the finite level spacing on the box.

${ }^{9}$ K. Le Hur, Phys. Rev. Lett. 92, 196804 (2004)

10 A. J. Leggett, S. Chakravarty, A. T. Dorsey, M. P. A. Fisher, A. Garg, and W. Zwerger, Rev. Mod. Phys. 59, 1 (1987).

11 P. Joyez, V. Bouchiat, D. Esteve, C. Urbina, and M. H. Devoret, Phys. Rev. Lett. 79, 1349 (1997).

12 G. Zaránd, G.T. Zimányi, and F. Wilhelm, Phys. Rev. B 62, 8137 (2000).

13 D.L. Cox and A. Zawadowski, Adv. Phys. 47, 599 (1998).
14 J. von Delft and H. Schoeller, Annalen Phys. 7, 225 (1998).

15 G. Zaránd and E. Demler, Phys. Rev. B 66, 024427 (2002); L. Zhu and Q. Si, Phys. Rev. B 66, 024426 (2002).

16 G. Yuval and P. W. Anderson, Phys. Rev. B 1, 1522 (1970).

17 The dimension $x$ of a local operator at a fixed point $\hat{O}$ is defined through the asymptotic decay of its correlation function $\left\langle\mathrm{T}_{\tau} \hat{O}(\tau) \hat{O}(0)\right\rangle \sim 1 / \tau^{2 x}$. Since these local operators live in one dimension (time), the scaling dimension of the corresponding dimensionless coupling in the Hamiltonian is simply $y=1-x$. [See e.g. J. Cardy, Scaling and Renormalization in Statistical Physics (Cambridge University Press, Cambridge, 1996)]

18 J.M. Kosterlitz, J. Phys. C 7, 1046 (1974).

19 G.T. Zimányi, K. Vladár, and A. Zawadowski, Phys. Rev. Lett 56, 286 (1986).

${ }^{20}$ K.G. Wilson, Rev. Mod. Phys, 47, 773 (1975).

21 T. A. Costi, A. Hewson, and V. Zlatic, J. Phys.: Condens. Matter 6, 2519 (1994).

${ }^{22}$ R. Bulla, T. A. Costi, and D. Vollhardt, Phys. Rev. B 64, 045103 (2001).

23 W. Hofstetter, Phys. Rev. Lett. 85, 1508 (2000)

24 R. Bulla N.-H. Tong, M. Vojta, Phys. Rev. Lett. 91, 170601 (2003)

25 R. Bulla, H.-J. Lee, N.-H. Tong, Matthias Vojta, cond-mat/0407559

26 T. A. Costi Phys. Rev. Lett. 80, 1038 (1998)

27 T. Costi, Phys. Rev. Lett. 85, 1504 (2000) 
28 G. Zaránd, L. Borda, J. von Delft, and N. Andrei, Phys. Rev. Lett. 93, 107204 (2004)

${ }^{29}$ C. Itzykson and J. B. Zuber, Quantum Field Theory
(McGraw-Hill, New York, 1985). 